\title{
Emergence of Lévy walks in systems of interacting individuals
}

DOI:

10.1103/PhysRevE.95.030107

\section{Document Version}

Final published version

Link to publication record in Manchester Research Explorer

\section{Citation for published version (APA):}

Fedotov, S., \& Korabel, N. (2017). Emergence of Lévy walks in systems of interacting individuals. Physical Review E, 95(3), [030107(R)]. https://doi.org/10.1103/PhysRevE.95.030107

\section{Published in:}

Physical Review E

\section{Citing this paper}

Please note that where the full-text provided on Manchester Research Explorer is the Author Accepted Manuscript or Proof version this may differ from the final Published version. If citing, it is advised that you check and use the publisher's definitive version.

\section{General rights}

Copyright and moral rights for the publications made accessible in the Research Explorer are retained by the authors and/or other copyright owners and it is a condition of accessing publications that users recognise and abide by the legal requirements associated with these rights.

\section{Takedown policy}

If you believe that this document breaches copyright please refer to the University of Manchester's Takedown Procedures [http://man.ac.uk/04Y6Bo] or contact uml.scholarlycommunications@manchester.ac.uk providing relevant details, so we can investigate your claim.

\section{OPEN ACCESS}




\title{
Emergence of Lévy walks in systems of interacting individuals
}

\author{
Sergei Fedotov and Nickolay Korabel \\ School of Mathematics, The University of Manchester, Manchester M13 9PL, United Kingdom
}

(Received 30 June 2016; published 28 March 2017)

\begin{abstract}
We propose a model of superdiffusive Lévy walk as an emergent nonlinear phenomenon in systems of interacting individuals. The aim is to provide a qualitative explanation of recent experiments [G. Ariel et al., Nat. Commun. 6, 8396 (2015)] revealing an intriguing behavior: swarming bacteria fundamentally change their collective motion from simple diffusion into a superdiffusive Lévy walk dynamics. We introduce microscopic mean-field kinetic equations in which we combine two key ingredients: (1) alignment interactions between individuals and (2) non-Markovian effects. Our interacting run-and-tumble model leads to the superdiffusive growth of the mean-squared displacement and the power-law distribution of run length with infinite variance. The main result is that the superdiffusive behavior emerges as a cooperative effect without using the standard assumption of the power-law distribution of run distances from the inception. At the same time, we find that the collision and repulsion interactions lead to the density-dependent exponential tempering of power-law distributions. This qualitatively explains the experimentally observed transition from superdiffusion to the diffusion of mussels as their density increases [M. de Jager et al., Proc. R. Soc. B 281, 20132605 (2014)].
\end{abstract}

DOI: 10.1103/PhysRevE.95.030107

Introduction. Lévy walk [1-3] is an important concept which describes a wide spectrum of physical and biological processes involving stochastic transport [4-10]. Cold atoms moving in dissipative optical lattices [11], endosomal active transport in living cells [12], and T-cells migrating in the brain tissue [13] are just several examples where Lévy walks were reported. It is argued that living organisms can use Lévy transport to accelerate pattern formation [14] and to optimize searching for sparse food [15-18]. Recently an intriguing behavior of swarming bacteria was found: they fundamentally change their collective motion from simple diffusion into a superdiffusive Lévy walk dynamics [19]. The extraordinary feature of this movement is that the emergence of a superdiffusive motility is a result of the interactions between bacteria rather than the standard mechanism of controlling the individual frequency of tumbling. However, it is still an open question how Lévy walks emerge in systems of interacting self-propelled particles. The current theory of Lévy walk [8] assumes noninteracting particles and power-law distribution of traveled distances from the inception.

The collective behavior of large groups of interacting individuals such as bird flocks, fish schools, and the collective migration of cells or bacteria is another rapidly growing area of active matter research [20-38]. There exist two main types of models used for a collective behavior: (1) Lagrangian models describing the movements of self-propelled particles in terms of nonlinear equations for the positions and velocities of all particles [20-23], and (2) kinetic models involving partial differential equations for the population densities [24-30]. In this Rapid Communication we are only concerned with the nonlinear kinetic and macroscopic equations. They have been used to describe interactions between individuals and investigate the formation of a large variety of spatiotemporal self-organized aggregations. Most of these models converge to the density-dependent diffusive transport and do not lead to non-Markovian Lévy walks.

In this Rapid Communication we propose a kinetic nonlinear Lévy walk model for interacting individuals in which the superdiffusion is an emerging collective phenomenon.
Importantly, we do not assume the power-law distribution of traveled distances or running times with infinite second moments from the inception. Motivated by the recent experiments [19,39], we introduce microscopic mean-field kinetic equations in which we combine two key ingredients: (1) the turning rate that depends on alignment and collision interactions between individuals and (2) non-Markovian effects. The crucial problem here is how to incorporate nonlinear interactions into non-Markovian superdiffusion. To implement nonlinear effects we use the structural density approach together with a population density-dependent turning rate. This method has been used by the authors for the analysis of subdiffusive random walks $[40,41]$ and Lévy walks $[42,43]$.

We take into account the interactions between walkers on the mesoscopic level, at which the turning rate nonlocally depends on the mean-field population density (nonlinear effect) and running time (non-Markovian effect). Our nonlinear non-Markovian persistent random-walk model explains the emergence of superdiffusive motion of bacteria within a swarm. At the same time we find that collision interactions lead to the transition from superdiffusion to the standard diffusion through nonlinear tempering of the power-law distribution of run distances. One should note that in this Rapid Communication we do not consider a Lévy flight, which is a simple Markovian random walk with a heavy-tailed step length distribution.

Previously, anomalous behavior of active systems involving superdiffusion was observed in Lagrangian [21,22] and hydrodynamic models [36,37]. In Lagrangian models [21,22] superdiffusive behavior is transient. In hydrodynamic models $[36,37]$ it was found that the spreading of active particles in the direction perpendicular to the direction of movement of the swarm is superdiffusive. We should note that these models do not correspond to a Lévy walk. They are fundamentally different from our model in which the dependence of the tumble (switching) rate on density and running time leads to a Lévy walk in the mean direction of movement of the swarm. Chepizhko and Peruani [44] found a subdiffusive motion of active particles in an environment of randomly distributed 
pointlike obstacles. These particles were interacting with obstacles which obstructed their motion and generated subdiffusive behavior similarly to the random Lorentz gas model [45]. Also, we should mention the one-dimensional (1D) models of active systems [38] and [46]. O'Loan and Evans [38] introduced the interaction of particles by assuming that the velocity of a particle is flipped with the probability which depends on the velocity of the majority of the particles at the site and its two nearest neighbors. They did not consider nonMarkovian effects and did not obtain anomalous superdiffusive behavior. Schnitzer [46] introduced a hyperbolic random-walk model in which a flipping (collision) rate depends on the direction of motion. The essential difference between our model and this chemotactic model is that the interactions of particles and anomalous behavior were not considered in [46].

Nonlinear Lévy walk model in $1 D$. We consider proliferating individuals moving either left or right along onedimensional space at a constant speed $v$. The key characteristic of such random movement is the turning rate $\mathbb{T}$, which defines moments when individuals change their direction of movements. The generalization for high dimensions is outlined in the Supplemental Material [47]. The detailed two-dimensional numerical simulations will be considered in a future work. Note that $1 \mathrm{D}$ is enough to show that the superdiffusion can be an emerging nonlinear phenomenon.

We introduce the structural densities of individuals, $n_{+}(x, t, \tau)$ and $n_{-}(x, t, \tau)$, at location $x$ and time $t$ that move in the right direction $(+)$ or the left direction $(-)$ during running time $\tau$ since the last velocity switching [48]. The mean density of individuals moving right, $(+)$, and left, $(-)$, are defined as

$$
\rho_{ \pm}(x, t)=\int_{0}^{\infty} n_{ \pm}(x, t, \tau) d \tau,
$$

and the total density $\rho(x, t)=\rho_{+}(x, t)+\rho_{-}(x, t)$.

To describe the random movement of individuals with interactions, we assume that the rate $\mathbb{T}_{ \pm}$at which individuals change their direction of motion depends not only on running time $\tau$ (non-Markovian effect) but also on the population densities $\rho_{+}$and $\rho_{-}$(nonlinear effect):

$$
\mathbb{T}_{ \pm}\left(\tau, \rho_{+}, \rho_{-}\right)=\frac{\mu_{ \pm}\left(\rho_{+}, \rho_{-}\right)}{\tau_{0}+\tau}+\gamma_{ \pm}\left(\rho_{\mp}\right)
$$

where $\tau_{0}$ is the time parameter. Inverse dependence of the first term in $\mathbb{T}_{ \pm}$on the running time $\tau$ leads to a strong persistence of the random walk. The function $\mu_{ \pm}\left(\rho_{+}, \rho_{-}\right)$describes the alignment effects leading to cooperative movement of individuals in one direction. If, for example, an individual moves to the right and senses that neighboring conspecifics move in the same direction, then the likelihood of velocity switching decreases. The positive function $\gamma_{ \pm}\left(\rho_{\mp}\right)$ takes into account the increase in the turning rate $\mathbb{T}_{ \pm}$when the individuals avoid the collisions with those moving in the opposite direction. The external forces or chemotactic factors could be included analogously to systems with subdiffusion $[49,50]$. Here we do not consider these effects.

In this Rapid Communication we model the alignment among individuals by the function

$$
\mu_{ \pm}\left(\rho_{+}, \rho_{-}\right)=\mu f\left(A_{ \pm}\right)
$$

with a nonlocal density-dependent function $A_{ \pm}(x, t)$ :

$$
A_{ \pm}=a \int_{\mathbb{R}} e^{-|z| / l_{a}}\left[\alpha \rho_{ \pm}(x+z, t)-\beta \rho_{\mp}(x+z, t)\right] d z .
$$

Here $\mu$ is the exponent of a power-law distribution in the absence of nonlinear interactions $\left(A_{ \pm}=0\right), f(x)$ is a positive and decreasing function of $x$ with $f(0)=1, a$ is the strength of interactions, and $\alpha, \beta$ are weight parameters. The decreasing function $f\left(A_{ \pm}\right)$indicates that the turning rate $\mathbb{T}_{ \pm}$is reduced due to the presence of many conspecifics moving in the same direction. This negative dependence plays the central role in the transition from a standard random walk to a Lévy walk. The kernel $\exp \left(-|z| / l_{a}\right)$ describes a strength of alignment per unit density with the distance $|z| ; l_{a}$ is the characteristic length of the interaction zone. To illustrate the effect of alignment, let us consider the case $\alpha=\beta=1$ for which the nonlocal function $A_{+}$for right-moving individuals can be rewritten in terms of the flux $J=v\left(\rho_{+}-\rho_{-}\right)$. We can write

$$
A_{+}=a v^{-1} \int_{\mathbb{R}} \exp \left(-\frac{|z|}{l_{a}}\right) J(x+z, t) d z,
$$

so the increase in the flux $J$ leads to an increase of alignment effects and a decrease of turning rate $\mathbb{T}_{+}$. This indicates the emergence of nonlinear persistence which, together with running time persistence, can generate superdiffusive behavior. Such modeling is in agreement with the observation that the motion of swarming bacteria is mostly governed by the collective flow of the bacteria and surrounding fluids [19]. Note that the advantage of moving in a large group in the same direction is very similar to the "peloton" phenomenon in a road bicycle race. Similar nonlocal dependencies of the turning rate on the population density has been successfully used to describe the animal spatial group patterns and bacterial swarming in terms of the hyperbolic and kinetic models [25,27-30].

The second term $\gamma_{ \pm}>0$ in the turning rate $\mathbb{T}_{ \pm}$, Eq. (2), describes the collision and repulsion effects. We assume the increase in the turning rate $\mathbb{T}_{ \pm}$when individuals tend to avoid collisions with many conspecifics moving in the opposite direction:

$$
\gamma_{ \pm}\left(\rho_{\mp}\right)=r \int_{0}^{\infty} \exp \left(-\frac{z}{l_{r}}\right) \rho_{\mp}(x \pm z, t) d z,
$$

where $l_{r}$ is the effective repulsion size, and $r$ is the strength of repulsion. The similar repulsion rate has been well used in the hyperbolic model [28] to obtain spatial patterns. In our non-Markovian model, the role of the collision and repulsion rate $\gamma_{ \pm}$is drastically changed. This term is responsible for the shift from the superdiffusive Lévy walk to diffusion as the density increases [39].

The nonlinear equations for the structural densities $n_{+}(x, t, \tau)$ and $n_{-}(x, t, \tau)$ can be written as

$$
\frac{\partial n_{ \pm}}{\partial t} \pm v \frac{\partial n_{ \pm}}{\partial x}+\frac{\partial n_{ \pm}}{\partial \tau}=-\mathbb{T}_{ \pm}\left(\tau, \rho_{+}, \rho_{-}\right) n_{ \pm}
$$

We use symmetrical initial conditions for which all individuals start to move with zero running time

$$
n_{ \pm}(x, 0, \tau)=\frac{\rho(x, 0)}{2} \delta(\tau)
$$


The zero running time condition $(\tau=0)$ includes the proliferation of the individuals:

$$
n_{ \pm}(x, t, 0)=\int_{0}^{t}\left[\mathbb{T}_{\mp}\left(\tau, \rho_{+}, \rho_{-}\right) n_{\mp}+k(\rho) n_{ \pm}\right] d \tau,
$$

where $k(\rho)$ is the density-dependent proliferation rate. This condition corresponds to the case when newborn individuals have zero running time. It is convenient to introduce the total turning rates defined as

$$
i_{ \pm}(x, t)=\int_{0}^{t} \mathbb{T}_{ \pm}\left(\tau, \rho_{+}, \rho_{-}\right) n_{ \pm}(x, t, \tau) d \tau .
$$

Differentiating (1) with respect to time $t$ together with (7) and (8) we derive the balance equations for the unstructured mean densities $\rho_{+}(x, t)$ and $\rho_{-}(x, t)$ :

$$
\frac{\partial \rho_{ \pm}}{\partial t} \pm v \frac{\partial \rho_{ \pm}}{\partial x}=-i_{ \pm}(x, t)+i_{\mp}(x, t)+k(\rho) \rho_{ \pm}
$$

(see Supplemental Material). For Lévy walks without interactions, one can find

$$
i_{ \pm}(x, t)=\int_{0}^{t} K(\tau) \rho_{ \pm}(x \mp v \tau, t-\tau) d \tau,
$$

where $K(\tau)$ is the memory kernel determined by its Laplace transform [42] $\hat{K}(s) \simeq \frac{1}{T}\left(1+A s^{\mu-1}\right)$ for $1<\mu<2$, as $s \rightarrow$ 0 ( $T$ is mean running time and $A$ is a constant). For the nonlinear case, the expressions for $i_{ \pm}$are not known. In what follows we use numerical simulations to obtain our results.

Emergence of superdiffusion. To focus on the collective movement and the underlying mechanism of the superdiffusive behavior of the walkers, we neglect the repulsion effects $(r \ll$ a). Proliferation is also not a necessary ingredient, therefore we consider nonproliferating walkers $[k(\rho)=0]$; although, numerical simulations show that proliferation increases the effect by raising the population density. Since the turning rate $\mathbb{T}_{ \pm}$, Eq. (2), depends on both residence time $\tau$ and time $t$ (indirectly through $\rho_{+}$and $\rho_{-}$), we cannot define the running time probability density function (PDF). It can only be done for the linear case when $f=1$. For this classical Lévy case the turning rate reads $\mathbb{T}(\tau)=\mu /\left(\tau_{0}+\tau\right)$ and the running time PDF $\psi(\tau)$ defined in the standard way $\psi(\tau)=\mathbb{T}(\tau) \exp \left[-\int_{0}^{\tau} \mathbb{T}(\tau) d \tau\right][51]$, becomes the power-law density:

$$
\psi(\tau)=\frac{\mu \tau_{0}^{\mu}}{\left(\tau_{0}+\tau\right)^{1+\mu}} .
$$

For $1<\mu<2$, this PDF has a finite first moment and infinite second moment. This case corresponds to anomalous subballistic superdiffusion for which the mean-squared displacement is $\left\langle x^{2}\right\rangle \sim t^{3-\mu}[1-3]$.

Importantly, for individuals interacting via alignment we consider $f \neq$ const and $\mu>2$ for which the system without interactions has standard long-time diffusive behavior: $\left\langle x^{2}\right\rangle \sim$ $t$ as $t \rightarrow \infty$ [curve (1) in Fig. 2]. We do not assume the anomalous running time PDF from the inception as is done for a classical theory of superdiffusive transport [1-3]. In our simulations we chose $l_{a}=1, \alpha=1$, and $\beta=0$ in Eq. (4), and an exponential interaction function $f\left(A_{ \pm}\right)=\exp \left(-A_{ \pm}\right)$. For $\alpha=\beta=1$ we obtain similar results. At $t=0$ we consider a uniform distribution of individuals in the interval $(-1,1)$.

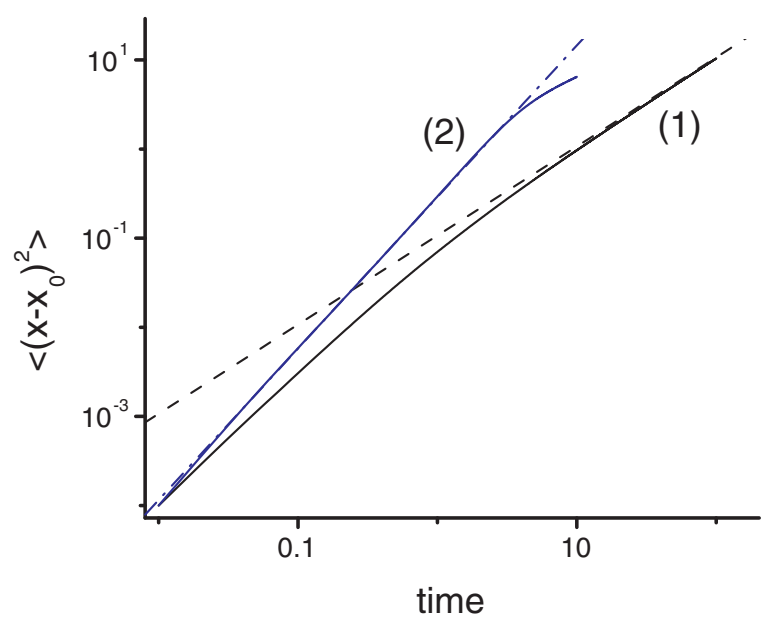

FIG. 1. Emergence of the Lévy walks when alignment dominates repulsion. The log-log plot of the MSD [curve (2)] displays the superdiffusive behavior with exponent 1.7 (dashed-dotted line). We have used $a=1.5, \alpha=1, \beta=0$, and $r=0$. Other parameters are $\mu=3, \tau_{0}=0.1, v=1$. Without interactions $a=0, r=0$ [curve (1)], the MSD grows linearly in the long-time limit (dashed line has the slope 1).

Figure 1 illustrates the emergence of the Lévy walk as the ensemble-averaged mean-squared displacement (MSD) [52] displays superdiffusive behavior [curve (2)]. For $\mu=3$ and $a=1.5$, we find $\left\langle\left(x-x_{0}\right)^{2}\right\rangle \simeq t^{1.7}$ (other parameters are listed in the figure caption). Since the individuals disperse in space, their density and therefore the strength of interactions decrease with time. That is, $f \rightarrow 1$ since $A_{ \pm} \rightarrow 0$. As a result, walkers perform normal diffusion at longer times [curve (2)]. Figure 2 confirms the emergence of the Lévy walk. It shows the power-law behavior of the run length PDF with exponent -2.7 for the same parameters used in Fig. 1. A typical trajectory of an individual involves long runs displaying anomalous persistence (walkers collectively move in one direction) [Fig. 3(b)]. The results of our model (Figs. 1 and 2) qualitatively explain the emergence of the Lévy walk observed experimentally for swarming bacteria [19]. We show that the standard switching (run-tumble) behavior of individuals is drastically changed due to a collective motion that facilitates the Lévy walk [19]. Interestingly, the alignment interactions lead to the creation of two groups of individuals called clumps that move to the left and to the right [Fig. 3(a)]. This is in agreement with the clumping behavior observed in nonlocal hyperbolic models for self-organized biological groups [29]. For small interaction strength $a \rightarrow 0$, there is no clumping phenomenon [Fig. 3(b)].

Nonlinear transition from superdiffusion to diffusion. We now ignore the alignment effects $\left[f\left(A_{ \pm}\right)=1\right]$ and focus on the repulsion and collision interactions. It follows from Eq. (2), the switching rate $\mathbb{T}_{ \pm}$is defined as

$$
\mathbb{T}_{ \pm}=\frac{\mu}{\tau_{0}+\tau}+\gamma_{ \pm}\left(\rho_{\mp}\right)
$$

where the interaction term $\gamma_{ \pm}$is defined in (5). Note that now we consider the case $1<\mu<2$. That is, without interactions we have a subballistic superdiffusive Lévy walk with powerlaw running time density, Eq. (11), and the MSD growing 


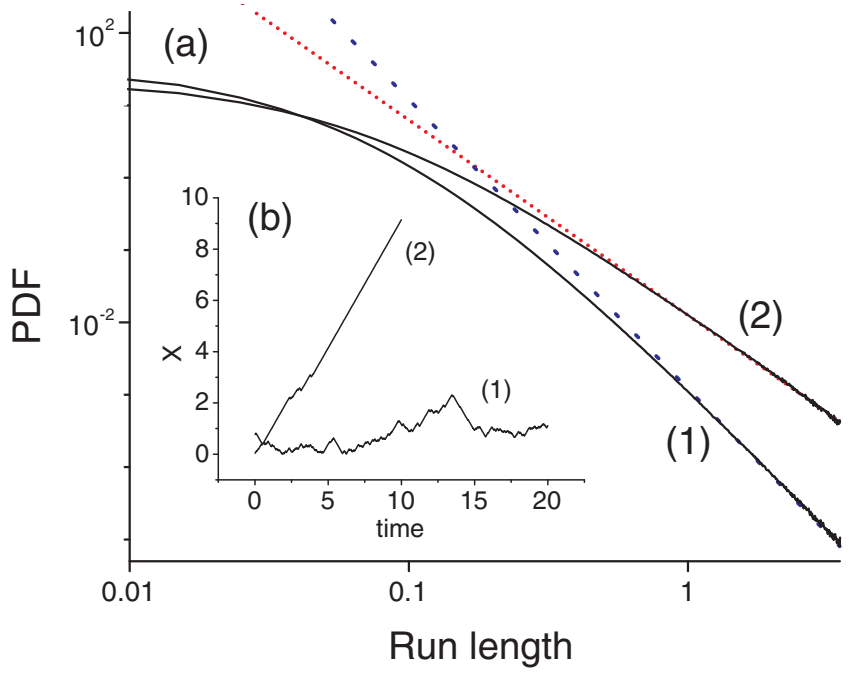

FIG. 2. (a) The run length PDF of the emerging Lévy walkers [curve (2)]. Parameters are the same as in Fig. 1. The run length PDF is a power law with the slope -2.7 (the dashed line), that is, the variance of the PDF is infinite. For noninteracting walkers the run length PDF is also a power law [curve (1)], but the slope is $-\mu-1=-4$ (the dotted line), so the variance is finite. (b) Typical trajectory of interacting Lévy walkers (curve (2); for better clarity we use $a=2.5$ ) is very persistent, unlike the Brownian-like trajectory for a walker without interactions [curve (1)].

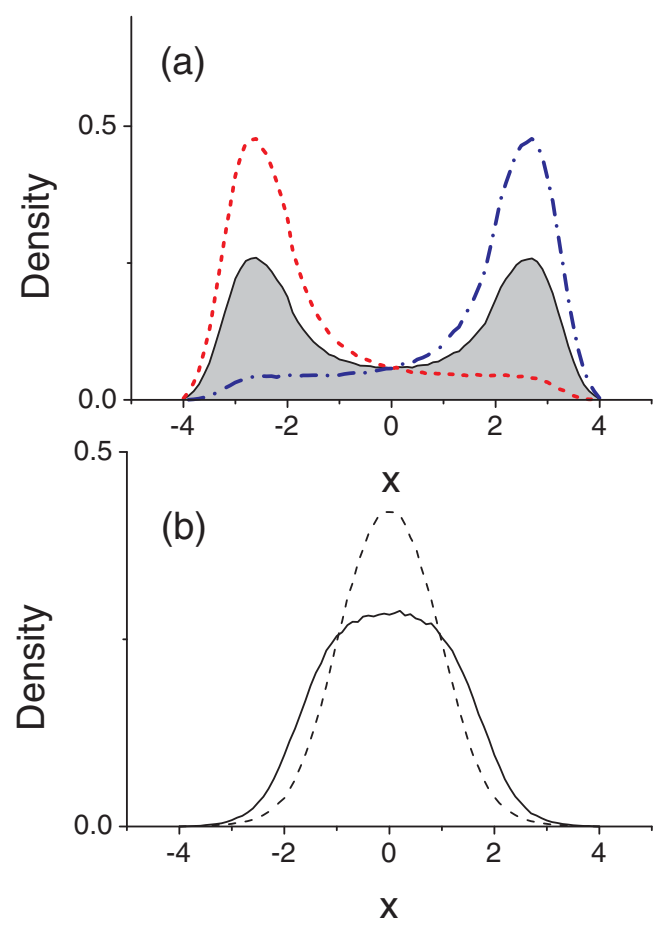

FIG. 3. (a) Alignment leads to the formation of two moving aggregates known as clumps. Density of the walkers $\rho$ (solid curve) develops two bumps corresponding to groups of walkers moving to the left $\rho_{-}$(dashed curve) and to the right $\rho_{+}$(dashed-dotted curve). Here $a=2.5$ and other parameters are the same as in Fig. 2. (b) For weak interactions, $a \rightarrow 0$, we find no Lévy walks and clumping behavior. The solid curve corresponds to $a=0.1$. Without interactions the density of walkers (dashed curve) is Gaussian apart from tails. All densities were calculated at $t=3$.

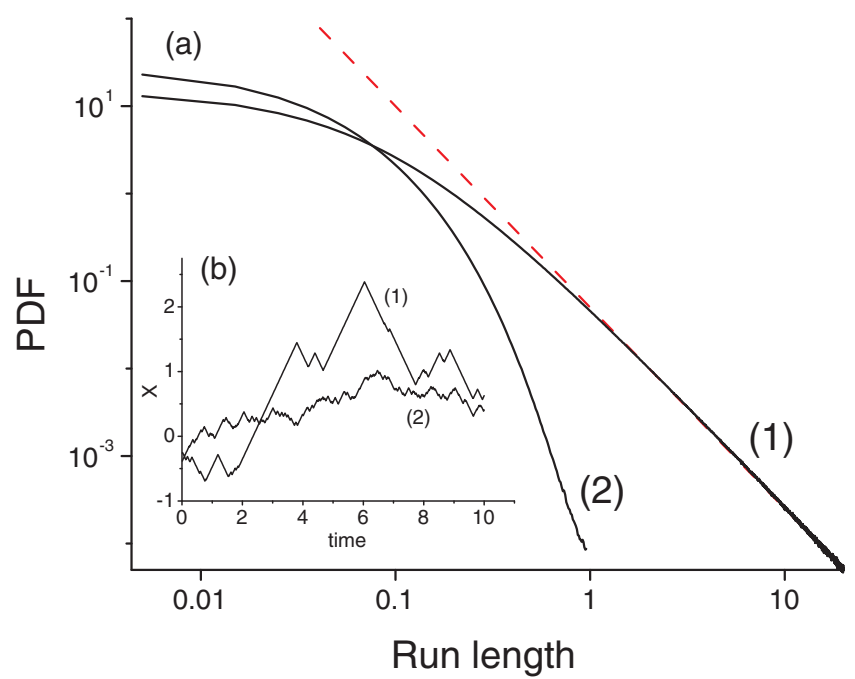

FIG. 4. (a) The run length PDF of individuals interacting repulsively. Interactions lead to the transition to Brownian diffusion. Here we use $r=10^{3}$ and $\mu=1.3$. Other parameters and the simulation procedure are the same. The run length PDF transitions from a power law with exponent $-\mu-1$ for the Lévy walk without interactions [curve (1)] to exponential distribution for the standard diffusion [curve (2)]. (b) Typical trajectories of walkers with interactions [curve (2)] and without interactions [curve (1)]. Repulsion interactions truncate the long runs of the Lévy walks.

as $\left\langle x^{2}\right\rangle \sim t^{3-\mu}$. We obtain explicit expressions for the total turning rates $i_{ \pm}$in terms of the density of walkers (for the derivation, see the Supplemental Material)

$$
\begin{aligned}
i_{ \pm}(x, t)= & \int_{0}^{t} K(t-\tau) \rho_{ \pm}(x \mp v(t-\tau), \tau) \\
& \times e^{-\int_{\tau}^{t} \gamma_{ \pm}\left(\rho_{\mp}(x \mp v(t-u), u)\right) d u} d \tau .
\end{aligned}
$$

It is clear from (12) that the rate $\gamma_{ \pm}$plays the role of a tempering parameter. This term is responsible for the shift of the superdiffusive Lévy walk toward standard diffusion as the density $\rho_{ \pm}$increases. The tempering effect of the repulsion and collision interactions is similar to the tempering due to the random death of walkers [42]. Figure 4 shows the results of numerical simulations corresponding to the rate (2) with $f\left(A_{ \pm}\right)=1$. In the absence of repulsion we consider a superdiffusive Lévy walk with $\mu=1.3$. A typical trajectory [Fig. 4(b)] has many long runs and the distribution of the run length is a power law with exponent $-\mu-1$ [curve (1) in Fig. 4(a)]. Repulsion and collision interactions drastically change the stochastic dynamics of individuals. The long runs are truncated and the trajectory appears Brownian [curve (2) in Fig. 4(b)]. The run length PDF becomes exponential [curve (2) in Fig. 4(a)], confirming the transition from a Lévy walk to Brownian diffusion. Such a transition was observed experimentally in the movement of mussels as their density increases [39].

Summary. We have proposed a nonlinear persistent randomwalk model of collectively moving individuals that interact via alignment and repulsion. The walkers' interactions have been taken into account on the mesoscopic level, at which the 
individuals' turning rate depends on the mean-field population density (nonlinear effect) and running time since the last velocity switching (non-Markovian effect). The main result of this Rapid Communication is that the nonlocal alignment leads to the anomalous nonlinear persistence of the random walkers and the emergence of the Lévy walk as a collective phenomenon. Importantly, this emergent superdiffusive movement of individuals is a nonlinear non-Markovian effect, and is not based on the standard assumption of a power-law running time distribution from the inception. We should note that non-Markovian effects are crucial, since the numerical simulations of the Markovian nonlinear model show no Lévy walks. We have qualitatively explained (1) the experimentally observed emergence of superdiffusive Lévy walks of swarming bacteria due to their collective dynamics and (2) the transition from subballistic superdiffusion to the Brownian motion of individuals interacting via repulsion and collision, which was observed in the movement of mussels as their density increases.

So far, most kinetic studies of a collective behavior have focused on spatiotemporal self-organized aggregations [24-30] and all of them do not deal with non-Markovian Lévy walks. Here, we propose an interacting run-and-tumble model where Lévy dynamics is an emergent collective phenomenon. In this way, we establish a link between the field of active matter and the field of anomalous Lévy transport which opens new possibilities in both areas of research.

Acknowledgment. This work was supported by EPSRC Grants No. EP/J019526/1 and No. EP/N018060/1.
[1] M. F. Shlesinger, B. J. West, and J. Klafter, Phys. Rev. Lett. 58, 1100 (1987).

[2] J. Klafter, A. Blumen, and M. F. Shlesinger, Phys. Rev. A 35, 3081 (1987).

[3] R. Metzler and J. Klafter, Phys. Rep. 339, 1 (2000).

[4] R. Klages, G. Radons, and I. M. Sokolov, Anomalous Transport: Foundations and Applications (Wiley-VCH Verlag, Weinheim, 2008).

[5] J. Klafter and I. M. Sokolov, First Steps in Random Walks: From Tools to Applications (Oxford University Press, Oxford, 2011).

[6] G. Margolin and E. Barkai, Phys. Rev. Lett. 94, 080601 (2005).

[7] A. Rebenshtok, S. Denisov, P. Hänggi, and E. Barkai, Phys. Rev. Lett. 112, 110601 (2014).

[8] V. Zaburdaev, S. Denisov, and J. Klafter, Rev. Mod. Phys. 87, 483 (2015).

[9] H. Stage, S. Fedotov, and V. Méndez, Math. Model. Nat. Phenom. 11, 157 (2016).

[10] J. P. Taylor-King, R. Klages, S. Fedotov, and R. A. Van Gorder, Phys. Rev. E 94, 012104 (2016).

[11] Y. Sagi, M. Brook, I. Almog, and N. Davidson, Phys. Rev. Lett. 108, 093002 (2012).

[12] K. Chen, B. Wang, and S. Granick, Nat. Mater. 14, 589 (2015).

[13] T. H. Harris, E. J. Banigan, D. A. Christian, C. Konradt, E. D. Tait Wojno, K. Norose, E. H. Wilson, B. John, W. Weninger, A. D. Luster, A. J. Liu, and C. A. Hunter, Nature (London) 486, 545 (2012).

[14] M. de Jager, F. J. Weissing, P. M. J. Herman, B. A. Nolet, and J. van de Koppel, Science 332, 1551 (2011).

[15] V. Mendez, D. Campos, and F. Bartumeus, Stochastic Foundations in Movement Ecology (Springer Science \& Business Media, New York, 2013).

[16] O. Beénichou, C. Loverdo, M. Moreau, and R. Voituriez, Rev. Mod. Phys. 83, 81 (2011).

[17] G. M. Viswanathan, S. V. Buldyrev, S. Havlin, M. G. E. da Luz, E. P. Raposo, and H. E. Stanley, Nature (London) 401, 911 (1999).

[18] F. Bartumeus, J. Catalan, U. L. Fulco, M. L. Lyra, and G. M. Viswanathan, Phys. Rev. Lett. 88, 097901 (2002).

[19] G. Ariel, A. Rabani, S. Benisty, J. D. Partridge, R. M. Harshey, and A. Beer, Nat. Commun. 6, 8396 (2015).
[20] H. Levine, W.-J. Rappel, and I. Cohen, Phys. Rev. E 63, 017101 (2000).

[21] H. Chaté, F. Ginelli, G. Grégoire, and F. Raynaud, Phys. Rev. E 77, 046113 (2008).

[22] C. Valeriani, M. Li, J. Novosel, J. Arlt, and D. Marenduzzo, Soft Matter 7, 5228 (2011).

[23] T. Vicsek and A. Zafeiris, Phys. Rep. 517, 71 (2012).

[24] J. Tailleur and M. E. Cates, Phys. Rev. Lett. 100, 218103 (2008).

[25] J. A. Carrillo, R. Eftimie, and F. K. O. Hoffmann, Kinet. Relat. Models 8, 413 (2015).

[26] F. D. C. Farrell, M. C. Marchetti, D. Marenduzzo, and J. Tailleur, Phys. Rev. Lett. 108, 248101 (2012).

[27] B. Pfistner, A one-dimensional model for the swarming behavior of myxobacteria, in Biological Motion, edited by W. Alt and G. Hoffmann (Springer-Verlag, Basel, 1989).

[28] R. Eftimie, G. de Vries, and M. A. Lewis, Proc. Natl. Acad. Sci. USA 104, 6974 (2007).

[29] R. C. Fetecau and R. Eftimie, J. Math. Biol. 61, 545 (2010).

[30] R. C. Fetecau, Math. Models Methods Appl. Sci. 21, 1539 (2011).

[31] A. Sokolov, I. S. Aranson, J. O. Kessler, and R. E. Goldstein, Phys. Rev. Lett. 98, 158102 (2007).

[32] A. Sokolov and I. S. Aranson, Phys. Rev. Lett. 109, 248109 (2012).

[33] A. Doostmohammadi, M. F. Adamer, S. P. Thampi, and J. M. Yeomans, Nat. Commun. 7, 10557 (2016).

[34] X.-L. Wu and A. Libchaber, Phys. Rev. Lett. 84, 3017 (2000).

[35] M. T. Butler, Q. Wang, and R. M. Harshey, Proc. Natl. Acad. Sci. USA 107, 3776 (2010).

[36] J. Toner and Y. Tu, Phys. Rev. E 58, 4828 (1998).

[37] J. Toner, H. Lowen, and H. H. Wensink, Phys. Rev. E 93, 062610 (2016)

[38] O. J. O’Loan and M. R. Evans, J. Phys. A: Math. Gen. 32, L99 (1999).

[39] M. de Jager, F. Bartumeus, A. Kölzsch, F. J. Weissing, G. M. Hengeveld, B. A. Nolet, P. M. J. Herman, and J. van de Koppel, Proc. R. Soc. B 281, 20132605 (2014).

[40] S. Fedotov, Phys. Rev. E 88, 032104 (2013).

[41] S. Fedotov and N. Korabel, Phys. Rev. E 92, 062127 (2015).

[42] S. Fedotov, A. Tan, and A. Zubarev, Phys. Rev. E 91, 042124 (2015). 
[43] S. Fedotov, Phys. Rev. E 93, 020101 (2016).

[44] O. Chepizhko and F. Peruani, Phys. Rev. Lett. 111, 160604 (2013).

[45] F. Hoefling and T. Franosch, Rep. Prog. Phys. 76, 046602 (2013).

[46] M. J. Schnitzer, Phys. Rev. E 48, 2553 (1993).

[47] See Supplemental Material at http://link.aps.org/supplemental/ 10.1103/PhysRevE.95.030107 for 2D generalization and transition from superdiffusion to diffusion.

[48] W. Alt, J. Math. Biol. 9, 147 (1980).
[49] S. Fedotov and N. Korabel, Phys. Rev. E 91, 042112 (2015).

[50] S. Fedotov, A. O. Ivanov, and A. Y. Zubarev, Math. Model. Nat. Phenom. 8, 28 (2013).

[51] D. R. Cox and H. D. Miller, The Theory of Stochastic Processes (Methuen, London, 1965).

[52] The ensemble-averaged MSD is calculated in a standard way: $\left\langle\left(x-x_{0}\right)^{2}\right\rangle=\sum_{i=1}^{N}\left[x_{i}(t)-x_{i}(0)\right]^{2} / N, x_{i}(t)$ is the position of the walker at time $t$ and $N=10^{6}$ is the number of walkers in the ensemble. 